\title{
The Association of Anesthetic Method With Developing Back Pain After Lower Extremity Operations
}

\author{
Marjan Joudi ${ }^{1}$; Mehdi Fathi ${ }^{1, *} ;$ Amin Dalili ${ }^{1} ;$ Saeed Jahanbakhsh ${ }^{2}$; Amir Merikhi Ardabili ${ }^{2}$; \\ Mohsen Akhondi ${ }^{2}$; Azra Izanloo ${ }^{3}$ \\ ${ }^{1}$ Surgical Oncology Research Center, Imam Reza Hospital, Faculty of Medicine, Mashhad University of Medical Sciences, Mashhad, Iran \\ ${ }^{2}$ Department of Anesthesiology, Imam Reza Hospital, Faculty of Medicine, Mashhad University of Medical Sciences, Mashhad, Iran \\ ${ }^{3}$ Department of Radiologic Technology, Faculty of Paraclinical Sciences, Mashhad University of Medical Sciences, Mashhad, Iran \\ ${ }^{*}$ Corresponding author: Mehdi Fathi, Surgical Oncology Research Center, Imam Reza Hospital, Faculty of Medicine, Mashhad University of Medical Sciences, Mashhad, Iran. Tel: +98- \\ 5118022677, Fax:+98-5138525209, E-mail: fathim@mums.ac.ir
}

Received: February 12, 2014; Revised: June 14, 2014; Accepted: July 19, 2014

\begin{abstract}
Background: Selection of anesthetic approach for lower extremity operations is often a controversial issue for anesthesiologists.
Objectives: The aim of this study was to compare the incidence and severity of back pain between general and spinal anesthesia, and to find effective factors in developing postoperative back pain.

Patients and Methods: In a randomized clinical trail, 148 patients with elective lower extremity surgeries were randomly allocated into two groups of 74. The first group received general anesthesia and the second group underwent spinal anesthesia. The incidence of back pain was observed and documented. The severity of back pain was assessed at the first, fourth and eighth postoperative weeks by visual analogue scale.

Results: The mean of patients' age was $35.50 \pm 13.34$ years. The incidence and mean of back pain severity among all participants were respectively $35.80 \%$ and $15.95 \%$ at the first week, $7.40 \%$ and $2.43 \%$ at the fourth week, and $1.4 \%$ and $0.27 \%$ at the eighth postoperative week. The incidence and mean of back pain severity in spinal group were respectively $39.2 \%$ and $18.11 \%$ at the first, $12.2 \%$ and $3.92 \%$ at the fourth, and $2.7 \%$ and $0.54 \%$ eighth postoperative weeks. In general anesthesia group, these figures were respectively $32.4 \%$ and $13.78 \%$ at the first week and $2.7 \%$ and $0.95 \%$ at the fourth postoperative week. No back pain was reported at the eighth postoperative week. The incidence and severity of back pain in the first week showed significant difference between the two groups, while the mean severity of back pain showed significant difference at the fourth week after operation.
\end{abstract}

Conclusions: Spinal anesthesia could be probably considered as the sole effective factor in the development of back pain after operation.

Keywords:Back Pain; Anesthesia; Lower Extremity

\section{Background}

Selection of anesthetic approach for lower extremity operations is often a controversial issue for anesthesiologists. Most of these patients are at two ends of the age spectrum: elderly with different types of underlying comorbidities at one end and young trauma patients with different types of injuries at the other end. In recent years, there has been a significant tendency towards the spinal anesthesia (SA) rather than general anesthesia (GA) in orthopedic surgeries. SA is a type of neuraxial blockade that involves the injection of local anesthetic drug with a long thin needle into the subarachnoid space (1). SA has been used since the late 19th century and now is the most common anesthetic technique of neural blockade through which a wide range of surgeries can be performed. Although GA has been the pioneer technique that induces the state of unconsciousness and sensory loss through the intravenous or inhaled agents, it is now less accepted by the anesthesiologists because of serious complications (2). In comparison to GA, SA may reduce the risk of many side effects including venous thromboembolism, intraoperative bleeding, cardiopulmonary problems, and postoperative analgesic requirements (3). Rhee et al. reported the dissatisfaction rate of 3.7\% for SA and suggested that patients' dissatisfaction and refusal of this method might be the result of their concerns about its possible complications (4). While headache is remarked as the most common side effect of SA, the incidence of complications varies widely (5). Although low back pain is often reported as a common postoperative complaint after both SA and GA, the association between anesthetic technique and back pain is still unclear. Some studies reported back pain as the most common postoperative complaints in patients who had SA, (6) while Schwabe et al. did not report such a complaint (7) and in the meantime, Quaynor et al. reported an equal incidence of back pain with GA and $S A(8,9)$. Although prior studies demonstrated the possible association between anesthesia and postoperative back pain, the association between GA or

Copyright (C) 2014, Iranian Society of Regional Anesthesia and Pain Medicine (ISRAPM); Published by Kowsar. This is an open-access article distributed under the terms of the Creative Commons Attribution-NonCommercial 4.0 International License (http://creativecommons.org/licenses/by-nc/4.0/) which permits copy and redistribute the material just in noncommercial usages, provided the original work is properly cited. 
SA and the incidence and severity of persistent back pain remains controversial. It is also unclear whether other concomitant factors can increase the incidence of back pain in these patients. Considering the advantages, safety, and potential complications of anesthetic procedure, the chosen technique might cause significant effects on both health and economic issues.

\section{Objectives}

This study was performed to compare the incidence and severity of back pain between GA and SA, and to explore factors affecting postoperative back pain.

\section{Patients and Methods}

The study was approved by the Ethics Committee of Mashhad University of Medical Sciences, Mashhad, Iran. We recruited 148 participants who underwent elective lower extremity surgeries in Orthopedic Department of Imam Reza Hospital of Mashhad between May 2011 and March 2012. Patients were randomly allocated to two equal groups of SA and GA. Inclusion criteria were as follows: patients aged between 18 and 60 years; ASA I or ASA II; elective surgery; and surgery duration in supine position shorter than three hours and in lateral position shorter than two hours. Exclusion criteria were as follows: any history of previous primary or referred back pain, back trauma, lumbar or thoracic vertebral surgery; any congenital abnormalities of the spine; body mass index $(\mathrm{BMI})>30 \mathrm{~kg} / \mathrm{m}^{2}$; any history of addiction; and presence of any transient neurological syndrome (TNS) such as pain or dysesthesia in one or both thighs, which radiated to the hip, thighs, or legs. Moreover, patients with systemic or local infection and those who received anticoagulant therapy were excluded from the study. An informed consent was obtained from all participants before the procedures. A sequential sampling method was used. Field data collection method was applied in which data collection tool was a questionnaire. By block randomization, patients were classified into two groups. The first group included 74 patients (mean age, $33.47 \pm 12.85$ years) who received GA with the same anesthetic protocol (Propofol, $2 \mathrm{mg} / \mathrm{kg}$; fentanyl, $4 \mu \mathrm{g}$ / $\mathrm{kg}$; cisatracurium, $0.2 \mathrm{mg} / \mathrm{kg}$, and maintenance of propofol, $100 \mu \mathrm{g} / \mathrm{kg} / \mathrm{min}$; and fentanyl, $50 \mu \mathrm{g}$ bolus per 20 minutes). The second group comprised 74 patients with the mean age of $37.53 \pm 13.61$ years who received SA, using $3 \mathrm{~mL}$ of $0.5 \%$ bupivacaine with the same procedure. The incidence of back pain was observed and documented. The severity of back pain was assessed at the first, fourth and eighth week of surgery via visual analogue scale (VAS), which ranged from zero to ten with zero standing for "no pain at all" and ten for "worst imaginable pain". After prepping and draping, with patient in sitting or lateral decubitus position, the $23,24,25$, or 27 gauge Quincke spinal needles (Dr. Japan Co, Tokyo, Japan) were used to access cerebrospinal fluid through the midline between L2-L3 or L3-L4 intervertebral space. Then $3 \mathrm{ml}$ of $0.5 \%$ bupivacaine (15 mg) (Mylan Co, France) at a rate of $0.2 \mathrm{~mL} / \mathrm{s}$ was injected. The number of puncture attempts was one to three times. Patients with more than three puncture attempts were excluded. Patients' data was collected using a questionnaire. Other data including patients' posture during anesthesia and surgery, duration of surgery and the number of puncture attempts to reach the subarachnoid space were documented as well. One week after surgery and at the first postoperative visit, the incidence and severity of back pain were evaluated and recorded. To determine their postoperative condition within a controlled period, we followed patients via telephone after hospital discharge at the fourth and eighth weeks of surgery. Data were analyzed using SPSS 11.5 (SPSS Inc, Chicago, IL, USA) with independent-samples t test, Mann-Whitney U, Chi square, and Fisher's exact tests.

\section{Results}

A total of 148 patients in two equal groups of 74 patients, comprised of 96 males (64.9\%) and 52 females (35.1\%) with the mean age of $35.50 \pm 13.35$ years, were recruited. The incidence of back pain for SA group was $39.2 \%, 12.2 \%$, and $2.7 \%$ at the first, fourth, and eighth postoperative weeks, respectively. The incidence of back pain for GA group was $32.4 \%$ and $2.7 \%$ at the first and the fourth weeks, respectively. No pain was reported at the eighth postoperative week in GA group. The incidence of back pain in the first postoperative week showed significant difference between two groups $(P=0.025)$, while the difference was not significant at the fourth $(\mathrm{P}=0.165)$ and eighth week $(P=0.497)$ (Table 1). Comparing the mean severity of back pain between two groups also showed no significant difference at the first and eighth weeks $(\mathrm{P}=0.156$ and $\mathrm{P}=$ 0.253 , respectively) but the difference was significant at the fourth postoperative week $(\mathrm{P}=0.030)$ (Table 1$)$. Our results showed that in the group with $\mathrm{GA}$, age $(\mathrm{P}=0.170)$, sex $(P=0.408)$, and BMI $(P=0.109)$ did not have any association with the severity of back pain at the first postoperative week. In addition, in the SA group, the age $(\mathrm{P}=$ 0.397), sex $(P=0.057)$, BMI $(P=0.423)$, surgery duration $(P=0.604)$, patient position during surgery $(P=0.526)$, the number of attempts for successful anesthesia $(\mathrm{P}=$ $0.338)$, and needle size $(P=0.209)$ did not show any association with the severity of back pain at the first postoperative week. The incidence of low back pain according to patient position, number of puncture attempts, and size of needle are respectively shown in Tables 2, 3, and 4. The association between probable factors and severity of back pain at the first postoperative week is shown in Table 5. There was no significant difference between the two groups with respect to the following variables: age $(\mathrm{P}$ $=0.071)$, sex $(P=0.863)$, surgical positioning $(P=0.688)$, weight $(\mathrm{P}=0.259)$, BMI $(\mathrm{P}=0.517)$, and surgery duration $(\mathrm{P}=0.21)$. No complications developed during or after the anesthetic procedures in this study. 
Joudi Met al.

Table 1. The Incidence and Mean Severity of Postoperative Back pain a,b

\begin{tabular}{lcccccc}
\hline & \multicolumn{2}{c}{ First Week } & \multicolumn{2}{c}{ Fourth Week } & \multicolumn{2}{c}{ Eighth Week } \\
\hline & Incidence & Mean Severity & Incidence & Mean Severity & Incidence & Mean Severity \\
\hline SA & 39.2 & 18.11 & 39.2 & 3.92 & 2.7 & 0.54 \\
GA & 32.4 & 13.78 & 2.7 & 0.95 & 0 & 0 \\
\hline
\end{tabular}

a Abbreviations: SA, spinal anesthesia; and GA, general anesthesia.

$\mathrm{b}$ Data are presented as \%.

Table 2. Incidence of Low Back Pain According to Position of Patient ${ }^{\mathrm{a}, \mathrm{b}}$

\begin{tabular}{lcc}
\hline Position & \multicolumn{2}{c}{ Incidence of Low Back Pain } \\
\hline GA & SA \\
\hline Prone & $22(91.7)$ & $27(93.1)$ \\
Lateral & $2(8.3)$ & $1(3.4)$ \\
\hline \multicolumn{2}{l}{ a Abbreviations: SA, spinal anesthesia; and GA, general anesthesia. } \\
b Data are presented as No. (\%).
\end{tabular}

Table 3. Incidence of Low Back Pain According to Number of Attempts ${ }^{a}$

\begin{tabular}{lc}
\hline Number of Attempt & Incidence of Low Back Pain \\
\hline $\mathbf{1}$ & 44.4 \\
$\mathbf{2}$ & 11.1 \\
$\mathbf{3}$ & 22.2 \\
$\mathbf{4}$ & 22.2 \\
\hline $\mathrm{a}$ Data are presented as \%.
\end{tabular}

Table 4. Incidence of Low Back Pain According to Size of Needle a

\begin{tabular}{lc}
\hline Size of Needle & Incidence of Low Back Pain \\
\hline 22 G & 6.9 \\
\hline 23 G & 20.7 \\
\hline 24 G & 37.9 \\
\hline 25 G & 31 \\
\hline 27 G & 2.2 \\
\hline a
\end{tabular}

${ }^{\mathrm{a}}$ Data are presented as $\%$.

Table 5. The Association of Probable Factors With Back Pain Severity at the First Postoperative Week the Studied Groups ${ }^{\text {a }}$

\begin{tabular}{lcc}
\hline Variables & \multicolumn{2}{c}{ Study Groups } \\
\hline Age, $\mathbf{y}$ & GA & SA \\
Male, $\%$ & $33.47 \pm 12.84$ & $37.53 \pm 13.61$ \\
BMI, kg/m & 66.2 & 63.5 \\
Weight, kg & $24.30 \pm 3.66$ & $24.78 \pm 3.4$ \\
Surgery Duration, min & $71.86 \pm 13.92$ & $74.38 \pm 12.73$ \\
Patient Position During Surgery & 0.267 & 0.526 \\
\hline $\begin{array}{l}\text { a Abbreviations: SA, spinal anesthesia; GA, general anesthesia; and BMI, } \\
\text { body mass index. }\end{array}$
\end{tabular}

\section{Discussion}

This study investigated the incidence and severity of postoperative back pain in GA and SA following elective lower extremity surgeries. The possible predisposing factors related to developing back pain following these procedures were also evaluated and compared between two groups. None of the abovementioned studied factors had a significant effect on developing back pain. Nevertheless, in SA group, the incidence of back pain at the first and the mean of back pain severity at the fourth postoperative weeks were significantly higher than that of GA group. In comparison with the study of Dahl et al. (10) and Gafari et al. (9), which showed the same incidence of back pain after both GA and SA, others such as Horlocker et al. (11) and Schwabe et al. (7) studies suspected the association between back pain and SA. Our findings showed higher incidence of back pain after SA. In accordance with this study, the results of Pinczower et al.(12) study demonstrated that back pain was more prevalent after SA. Various factors including trauma to the ligament, paraspinal muscle spasms, and ligamentous tension during changing position in a patient with skeletal muscle relaxation have been mentioned as causes of higher incidence of back pain after SA (13). In Edomwonyi and Isesele study on 120 patients, back pain was noted as a part of the neurological syndrome with an incidence of $10 \%$ for this syndrome and $6.6 \%$ for low back pain, (14) while the current study showed an incidence of $35.80 \%$ for back pain after the first week of surgery. In contrast to our study, investigation of Quaynor et al. in 2001 on 106 patients under SA revealed that none of the patients complained of back pain (8). While some studies demonstrated different incidence of postoperative back pain, ranging from $2 \%$ to $26 \%$, in the study of Schwabe et al. $56 \%$ of the patients complained of back pain five days after surgery and this figure decreased to $12.3 \%$ in the third month of follow-up (7). Nonetheless, with regard to different time of pain assessment, our study indicated lower incidence of postoperative back pain. It is worth noting that patients with history of back pain before SA were not excluded from Schwabe et al. study (7). According to Sarma et al., 10.2\% of patients after SA experienced back pain, which showed no statistically significant association between size of the needle as well as number of the attempts to perform the procedure and incidence of low back pain (15). In another study, Breivik et al. found that needle size did not affect headache and low back pain intensity after SA (16). In another study, the incidence of 
low back pain in spinal group was not significantly higher than control group in which normal vaginal delivery was done without any intervention (17). These results are in accordance with our findings. According to the literatures, all the studies did not specifically deal with the factors influencing the incidence of back pain after SA (7). In conclusion, SA is an easy, effective, reliable, and economically affordable method of anesthesia and it has been widely implemented in various surgeries due to less serious complications and more patients' satisfaction in comparison to GA. This study suggests that SA could be considered as an effective factor in developing back pain after orthopedic surgeries in the absence of back pain history. However, it indicates that anesthetic method cannot justify the severity of postoperative back pain. Hence, more studies are needed to determine and control other effective factors in severity of back pain after various surgeries and in different medical conditions.

\section{Acknowledgements}

The results described in this paper were part of a thesis submitted by the fifth author for a postgraduate degree in anesthesiology. We sincerely acknowledge Ms. M. Hassanpour for editing the manuscript.

\section{Authors' Contributions}

Design: Marjan Joudi, Mehdi Fathi, and Amir Merikhi Ardabili; definition of intellectual content: Mehdi Fathi and Amir Merikhi Ardabili; literature search: Mehdi Fathi, Amin Dalili, and Amir Merikhi Ardabili; data acquisition: Mehdi Fathi, Amin Dalili, and Amir Merikhi Ardabili; data analysis: Amir Merikhi Ardabili and Mohsen Akhondi; statistical analysis: Mohsen Akhondi; manuscript preparation: Mehdi Fathi, Amin Dalili, Saeed Jahanbakhsh, Mohsen Akhondi, and Azra Izanloo; manuscript editing: Mehdi Fathi, Amin Dalili, Saeed Jahanbakhsh, Mohsen Akhondi, and Azra Izanloo; manuscript review: Marjan Joudi, Mehdi Fathi, Amin Dalili, Saeed Jahanbakhsh, Amir Merikhi Ardabili, Mohsen Akhondi, and Azra Izanloo.

\section{Funding/Support}

The study was supported by the Vice Chancellor for Re- search of Mashhad University of Medical Sciences.

\section{References}

1. Brown DS. Epidural and Cudal Anesthesia. In: Miller RD, Eriksson LI, Fleisher LA, Wiener Kronish JP,, Young WL editors. Miller's Anesthesia. 7th ed. California: Churchil Livingstone;2010. pp.1611-38.

2. Gonano C, Leitgeb U, Sitzwohl C, Ihra G, Weinstabl C, Kettner SC. Spinal versus general anesthesia for orthopedic surgery: anesthesia drug and supply costs. Anesth Analg. 2006;102(2):524-9.

3. Raw DA, Beattie JK, Hunter JM. Anaesthesia for spinal surgery in adults. Br J Anaesth. 2003;91(6):886-904.

4. Rhee WJ, Chung CJ, Lim YH, Lee KH, Lee SC. Factors in patient dissatisfaction and refusal regarding spinal anesthesia. Korean J Anesthesiol. 2010;59(4):260-4.

5. Turnbull DK, Shepherd DB. Post-dural puncture headache: pathogenesis, prevention and treatment. Br J Anaesth. 2003;91(5):71829.

6. Pittoni G, Toffoletto F, Calcarella G, Zanette G, Giron GP. Spinal anesthesia in outpatient knee surgery: 22-gauge versus 25-gauge Sprotte needle. Anesth Analg. 1995;81(1):73-9.

7. Schwabe K, Hopf HB. Persistent back pain after spinal anaesthesia in the non-obstetric setting: incidence and predisposing factors. BrJAnaesth. 2001;86(4):535-9.

8. Quaynor H, Corbey M, Berg P. Spinal anaesthesia in day-care surgery with a 26-gauge needle. BrJ Anaesth. 1990;65(6):766-9.

9. Ghafari MH, Movafegh A, Sadri Zadeh N. A Comparison of Incidence of the Postdural Puncture Headache and Low Back Pain in the Cesarean Section Patients Undergoing Spinal Anesthesia and General Anesthesia: A Randomized Clinical Trial Study. Res J Biol Sci. 2009;4(2):187-9.

10. Dahl JB, Schultz P, Anker-Moller E, Christensen EF, Staunstrup HG, Carlsson P. Spinal anaesthesia in young patients using a 29-gauge needle: technical considerations and an evaluation of postoperative complaints compared with general anaesthesia. BrJ Anaesth. 1990;64(2):178-82.

11. Horlocker TT, McGregor DG, Matsushige DK, Schroeder DR, Besse JA. A retrospective review of 4767 consecutive spinal anesthetics: central nervous system complications. Perioperative Outcomes Group. Anesth Analg. 1997;84(3):578-84.

12. Pinczower GR, Gyorke A. Vertebral osteomyelitis as a cause of back pain after epidural anesthesia. Anesthesiology. 1996;84(1):215-7.

13. Holder HG, Graves CL. Spinal anesthesia; a survey of neurologic complications. Calif Med.1955;82(2):426-9.

14. Edomwonyi NP, Isesele TO. Transient neurological symptoms following spinal anesthesia for cesarean section. Middle East J Anesthesiol. 2010;20(6):809-13.

15. Sarma VJ, Lundstrom J. Epidural anaesthesia for day care surgery. A retrospective study. Anaesthesia.1989;44(8):683-5.

16. Breivik EK, Bjornsson GA, Skovlund E. A comparison of pain rating scales by sampling from clinical trial data. Clin J Pain. 2000;16(1):22-8.

17. Lak M, Forozanmehr M, Dodange F. Spinal versus epidural anesthesia complications, outcomes and pain relieving capacity. Trauma Mon. 2008;13(2):153-61. 